\title{
ON THE LOCATION OF THE ROOTS OF THE JACOBIAN OF TWO BINARY FORMS, AND OF THE DERIVATIVE OF A

\author{
RATIONAL FUNCTION*
}

BY

\section{J. L. WALSH}

\section{INTRODUCTION}

Professor Bôcher has shown how the roots of certain algebraic invariants can be determined as the positions of equilibrium in the field of force due to properly situated repelling and attracting particles. $\dagger \mathrm{He}$ considers a number of fixed particles either in a plane or on the surface of a sphere (the stereographic projection of the plane) and each of these particles is supposed to repel with a force equal to its mass divided by the distance. If a particle has negative mass, it attracts instead of repelling. The plane of the particles can be considered as the Gauss plane, and with this convention Bôcher proves the following theorem: $\ddagger$

Theorem I. The vanishing of the jacobian of two binary forms $f_{1}$ and $f_{2}$ of degrees $p_{1}$ and $p_{2}$ respectively determines the points of equilibrium in the field of force due to $p_{1}$ particles of mass $p_{2}$ situated at the roots of $f_{1}$, and $p_{2}$ particles of mass $-p_{1}$ situated at the roots of $f_{2}$.

Perhaps it is desirable briefly to indicate the proof of this theorem. We give the proof merely for the plane field. Let fixed particles of masses $m_{1}$, $m_{2}, \cdots, m_{n}$ be placed at the points represented by the complex quantities $e_{1}, e_{2}, \cdots, e_{n}$ respectively. Then at any point $x$ of the plane, the force due to these fixed particles is in magnitude, direction, and sense

$$
K\left(\frac{m_{1}}{x-e_{1}}+\frac{m_{2}}{x-e_{2}}+\cdots+\frac{m_{n}}{x-e_{n}}\right),
$$

where the symbol $K$ indicates the conjugate of the complex quantity following.

* Presented to the Society, February 23, 1918.

† Maxime Bôcher, $A$ problem in statics and its relation to certain algebraic invariants, P $\mathrm{r}$ o ceedings of the American Academy of Arts and Sciences, vol. 40 (1904), p. 469. I am indebted to Professor Bôcher for a number of suggestions concerning the present paper.

$\ddagger$ L. c., p. 476 . 
If homogeneous variables are introduced by the formulas

$$
x=\frac{x_{1}}{x_{2}}, \quad e_{i}=\frac{e_{i}^{\prime}}{e_{i}^{\prime \prime}},
$$

the plane field described in Theorem I becomes

$$
K\left[x_{2}\left(p_{2} \sum_{i=1}^{i=p_{1}} \frac{e_{i}^{\prime \prime}}{e_{i}^{\prime \prime} x_{1}-e_{i}^{\prime} x_{2}}-p_{1} \sum_{i=p_{1}+1}^{i=p_{1}+p_{2}} \frac{e_{i}^{\prime \prime}}{e_{i}^{\prime \prime} x_{1}-e_{i}^{\prime} x_{2}}\right)\right]
$$

$$
=K\left[x_{2}\left[p_{2} \frac{\frac{\partial f_{1}}{\partial x_{1}}}{f_{1}}-p_{1} \frac{\partial f_{2}}{\partial x_{1}}\right]\right]
$$

where

$$
\begin{aligned}
& f_{1}=\left(e_{1}^{\prime \prime} x_{1}-e_{1}^{\prime} x_{2}\right) \cdots\left(e_{p_{1}}^{\prime \prime} x_{1}-e_{p_{1}}^{\prime} x_{2}\right) \\
& f_{2}=\left(e_{p_{1}+1}^{\prime \prime} x_{1}-e_{p_{1}+1}^{\prime} x_{2}\right) \cdots\left(e_{p_{1}+p_{2}}^{\prime \prime} x_{1}-e_{p_{1}+p_{2}}^{\prime} x_{2}\right) .
\end{aligned}
$$

The quantity in the brackets in (1) reduces to the quotient of the jacobian of $f_{1}$ and $f_{2}$ by $f_{1} f_{2}$, when Euler's theorem for homogeneous functions is applied. This completes the proof of Theorem I. It is to be noted that the jacobian vanishes not only at the points of no force, but also at the multiple roots of either form or a common root of the two forms; such a point is called a point of pseudo-equilibrium.

From the mechanical interpretation of Theorem I, Bôcher derives a number of results concerning the location of the roots of the jacobian with reference to the location of the roots of the ground forms.*

When we consider the mechanical system, it is intuitively obvious that there can be no position of equilibrium very near any of the fixed particles. In the Corollary to Theorem II of the present paper there is determined explicitly (and in an infinite variety of ways) a circle which can be drawn separating any one of these particles from the roots of the jacobian. If we have not one fixed particle but $k$ particles, all attracting or all repelling, and if the remaining particles in the plane (or on the sphere) are sufficiently removed from those, then the mechanical system would lead us to expect that there could be no roots of the jacobian outside of and very near to a circle surrounding the $k$ particles. This is a rough indication of the considerations that lead to Theorem II.

In the latter part of the paper some applications of these results are made to the roots of the derivative of a rational function.

\footnotetext{
${ }^{*}$ See, e. g., Theorem III below.
} 


\section{PART I}

Let us consider the statical system in the plane due to fixed particles of the kind described. We shall make use of two lemmas, which, indeed, are more general than is necessary for our use.

Lemma I. If $Q$ is a point exterior to the circle $C$ whose center is $O$, then of all possible positions for a unit (repelling or attracting) particle on or within $C$, that position nearest to $Q$ causes the particle to exert the greatest force at $Q$,greatest not only in magnitude but also in component along $Q O$. That position farthest from $Q$ causes the particle to exert the least force at $Q$,- -least not only in magnitude but also in component along $Q O$.

Lemma II. If $Q$ is a point interior to the circle $C$ whose center is $O$, then of all possible positions for a unit (repelling or attracting) particle on or outside of $C$, that position nearest to $Q$ causes the particle to exert the greatest force at $Q$,greatest not only in magnitude but also in component along QO. Of all possible positions for an attracting particle on or outside of $C$, that position on $C$ which is farthest from $Q$ causes the particle to exert the force at $Q$ which has the greatest component in the direction and sense $Q O$.

The truth of each of these lemmas becomes evident upon inverting $C$ in the circle of unit radius and center $Q$, noting that the force exerted at $Q$ by a unit particle at $R$ is in direction and magnitude $R^{\prime} Q$, where $R^{\prime}$ is the inverse of $R$ in the unit circle whose center is $Q$.

We shall now apply these lemmas to Theorem I. Suppose there is in the plane a circle $C_{1}$ which contains on or within its circumference $k$ roots of $f_{1}$. Suppose there is a circle $C_{2}$-larger than $C_{1}$ and concentric with itoutside of which lie all the remaining $p_{1}-k$ roots of $f_{1}$. Suppose further that there is a circle $C_{3}$-also larger than $C_{1}$ and concentric with it-outside of which lie all the roots of $f_{2}$. Then we shall try to determine a circle $C_{0}$ larger than $C_{1}$ and concentric with it and such that there is no root of the jacobian of $f_{1}$ and $f_{2}$ within the annular region between $C_{0}$ and $C_{1}$.

We denote by $O$ the common center of $C_{1}, C_{2}$, and $C_{3}$, and the radii of these circles by $a, b$, and $c$, respectively. We have supposed that $a<b, a<c$. Set up the statical system of Theorem I and consider the force at a point $Q$ between $C_{1}$ and the smaller of $C_{2}$ and $C_{3}$. The component in the direction and sense $O Q$ of the force due to the $k$ positive particles (each of mass $p_{2}$ ) on or within $C$ is not less than $p_{2} k /(a+r)$, where $r$ is the distance $O Q$. The component in the direction and sense $Q O$ of the force due to the positive particles outside of $C_{2}$ (whose mass is $\left(p_{1}-k\right) p_{2}$ ) is not greater than $\left(p_{1}-k\right) p_{2} /(b-r)$. The component in the direction and sense $Q O$ of the negative particles outside of $C_{3}$ (whose mass is $-p_{1} p_{2}$ ) is not greater than 
$p_{1} p_{2} /(c+r)$. If $Q$ is a point of equilibrium, we must have

$$
\begin{gathered}
\frac{p_{2} k}{a+r} \leqq \frac{\left(p_{1}-k\right) p_{2}}{b-r}+\frac{p_{1} p_{2}}{c+r}, \\
\frac{k b c-p_{1} a b-\left(p_{1}-k\right) a c}{\left(p_{1}-k\right) b+p_{1} c-k a} \leqq r .
\end{gathered}
$$

If the left-hand member of (3) is positive, we construct the circle with that radius and center $O$, and denote this circle by $C_{0}$. Then it is readily seen that $C_{0}$ always lies within $C_{2}$ (unless $k=p_{1}$, when $C_{0}$ and $C_{2}$ coincide), and $C_{0}$ may or may not lie within $C_{1}$ and may or may not lie within $C_{3}$. If $C_{0}$ lies outside of $C_{1}$ but within $C_{3}$, then we have shown that the annular region between $C_{0}$ and $C_{1}$ contains no point of equilibrium. This region contains no root of either form and therefore no possible point of pseudo-equilibrium. Hence the annular region contains no root of the jacobian of the forms. If on the other hand, $C_{0}$ lies outside of $C_{3}$, then between $C_{1}$ and $C_{3}$ there is no root of the jacobian of the forms.

If $C_{0}$ lies outside of $C_{1}$, it is readily shown that there are precisely $k-1$ roots of the jacobian on or within $C_{1}$. Let the $k$ roots of $f_{1}$ that are on or within $C_{1}$ move continuously so as to coincide at the point $O$, while the other roots of $f_{1}$ and all the roots of $f_{2}$ remain fixed. If $Q$ is a position of equilibrium, inequality (2) obtains whenever $Q$ is anywhere within $C_{1}$. Hence by (3) there is no root of the jacobian within $C_{1}$ except at $O$; and $O$ is a $(k-1)$-fold root. During the change of the $k$ roots of $f_{1}$ the roots of the jacobian change continuously (at least when we refer to the sphere instead of the plane) and are never in the annular region between $C_{1}$ and the nearer of $C_{0}$ and $C_{3}$. Hence at the start there were just $k-1$ roots of the jacobian on or within $C_{1}$.

Let us determine the circle $C_{0}$ by invariant elements. Suppose a line through $O$ cuts the circles $C_{i}$ in the points $C_{i}^{\prime}$ and $C_{i}^{\prime \prime}(i=0,1,2,3)$ where the notation is such that $O$ separates no pair of points $C_{i}^{\prime}, C_{j}^{\prime}$. We find that*

$$
\left(C_{1}^{\prime \prime}, C_{2}^{\prime}, C_{3}^{\prime \prime}, C_{0}^{\prime}\right)=p_{1} / k \text {. }
$$

Hence, for the special case that $C_{1}, C_{2}$, and $C_{3}$ are concentric, with $C_{1}$ in the interior of $C_{2}$ and $C_{3}$, we have proved:

ThEOREM II. Suppose that $f_{1}$ and $f_{2}$ are two binary forms, the degree of $f_{1}$ being $p_{1}$, and suppose there are $k$ roots of $f_{1}$ which lie in a closed region $T_{1}$ bounded by a circle $C_{1}$. Suppose there is a second closed region $T_{2}$ bounded by a circle $C_{2}$,

* We are using the following definition for the cross-ratio:

$$
\left(z_{1}, z_{2}, z_{3}, z_{4}\right)=\frac{\left(z_{1}-z_{2}\right)\left(z_{3}-z_{4}\right)}{\left(z_{2}-z_{3}\right)\left(z_{4}-z_{1}\right)} .
$$


that $T_{2}$ has no point in common with $T_{1}$, and that $T_{2}$ contains the remaining $p_{1}-k$ roots of $f_{1}$. Suppose further that there is a third closed region $T_{3}$ bounded by a circle $C_{3}$ coaxial with $C_{1}$ and $C_{2}$, that $T_{3}$ has no point in common with $T_{1}$, and that $T_{3}$ contains all the roots of $f_{2}$.

1. If the circle $C_{0}$ described below lies in the region between $C_{1}$ and $C_{3}$, then there are no roots of the jacobian of $f_{1}$ and $f_{2}$ in the region included between $C_{1}$ and $C_{0}$; furthermore, there are just $k-1$ roots of the jacobian in $T_{1}$.

2. If the circle $C_{0}$ lies in the region between $C_{2}$ and $C_{3}$, then there are no roots of the jacobian of $f_{1}$ and $f_{2}$ in the region included between $C_{1}$ and $C_{3}$; moreover, there are just $k-1$ roots of the jacobian in $T_{1}$.

In this theorem, $C_{0}$ denotes that circle of the coaxial family to which $C_{1}, C_{2}$, and $C_{3}$ belong which is the locus of points $C_{0}^{\prime}$ such that

$$
\left(C_{1}^{\prime \prime}, C_{2}^{\prime}, C_{3}^{\prime \prime}, C_{0}^{\prime}\right)=p_{1} / k \text {. }
$$

$C_{i}^{\prime}$ and $C_{j}^{\prime \prime}$ denote the points in which any circle $T$ orthogonal to the circles of the family cuts the circle $C_{i}$, and the notation is such that on $T$, neither null circle of the family shall separate any of the pairs of points $C_{i}^{\prime}, C_{j}^{\prime}(i, j=0,1,2,3) .^{*}$

This theorem is proved for the case that $C_{1}, C_{2}$, and $C_{3}$ are not concentric by making a linear transformation that transforms them into concentric circles, with $C_{1}$ in the interior of $C_{2}$ and $C_{3}$. (Such a transformation always exists.) Since the theorem is true for this particular case, and since everything used in the theorem is invariant under linear transformation, the theorem is true as stated. $\dagger$

It is also true that Theorem II refers to the sphere as well as the plane, for everything essential in the theorem is invariant under stereographic projection.

* Reference to the italicized sentence immediately below (3) will show that 1 and 2 cannot occur at the same time. It may occur that $C_{0}$ lies in neither of these positions, which in the case of concentric circles means that $C_{0}$ lies within $C_{1}$; if this is true, the theorem makes no statement about the roots of the jacobian. Also, the circle $C_{0}$ may not exist, which means that the left-hand side of (3) is negative.

If no root of $f_{1}$ lies on $C_{1}$, if no root of $f_{1}$ lies on $C_{2}\left(k \neq p_{1}\right)$, or if no root of $f_{2}$ lies on $C_{3}$, then in case (1) no root of the jacobian can lie on $C_{0}$. This is immediately seen by omitting the equality sign in (2) and hence in (3).

Of course, a theorem similar to II can be proved for Bôcher's covariant $\phi$ (l. c., p. 474).

Theorem II can be applied to the roots of special types of polynomials, but as Professor Curtiss pointed out to me, the following more general theorem can be proved by means of Lemma II. This more general theorem is a special case of the theorem just suggested concerning the covariant $\varphi$.

If $f(z)$ is a polynomial of degree $n$ all of whose roots lie outside of a circle whose center is the origin and radius $b$, and if $k_{1}$ and $k_{2}$ are any positive numbers, then all the roots of $k_{1} z f^{\prime}(z)$ $-k_{2} f(z)$ lie outside the smaller of the two circles whose common center is the origin and whose radii are $b$ and $k_{2} b /\left(n k_{1}-k_{2}\right)$ respectively. See Laguerre, Euvres, vol. I, pp. 56, 133; see also the reference to Gonggrÿp below.

+ We consider the exterior of a circle, including the boundary and the point at infinity, to be a closed region. 
It is readily shown that Theorem II gives in general the largest region which will be free from roots of the jacobians of all pairs of forms which satisfy the hypothesis. Let us take the circles $C_{1}, C_{2}$, and $C_{3}$ in their original (concentric) positions, and first suppose $C_{0}$ to lie between $C_{1}$ and $C_{3}$. Then reference to inequality (2) shows that if $k \neq p_{1}$, the position of the particles which determine the field of force can be chosen so that (2) becomes an equality, and there will be a position of equilibrium on $C_{0}$. If $k=p, C_{0}$ and $C_{2}$ coincide, and we can consider $C_{2}$ to coincide with $C_{3}$. In this case, or if on the other hand $C_{0}$ lies outside of $C_{3}$, there can be chosen on $C_{3}$ a multiple root of $f_{2}$, which will be a root of the jacobian.

If we take $C_{1}$ a null circle $P$, and if we let $C_{2}$ and $C_{3}$ coincide and denote this circle by $C$, we have the following result:

Corollary. Suppose that $f_{1}$ and $f_{2}$ are two binary forms, the degree of $f_{1}$ being $p_{1}$, and suppose that the circle $C$ separates $P$ (a k-fold root of $\left.f_{1}\right)$ from those roots of $f_{2}$ and $f_{1}$ (other than $P$ ) which do not lie on $C$ itself. Then the circle $C_{0}$ separates $P$ from those roots of the jacobian of $f_{1}$ and $f_{2}$ (other than $P$ ) which do not lie on $C_{0}$ itself, where $C_{0}$ is that circle of the coaxial family determined by $C$ and $P$ which is the locus of points $C_{0}^{\prime}$ such that

$$
\left(P, C^{\prime}, C^{\prime \prime}, C_{0}^{\prime}\right)=p_{1} / k
$$

$C^{\prime}$ and $C^{\prime \prime}$ denote the intersections of $C$ with the circle through $P$ and $C_{0}^{\prime}$ orthogonal to $C$.

In the corollary, it is of course true that $C_{0}$ lies between $P$ and $C$ unless $k=p_{1}$, when $C_{0}$ and $C$ coincide.

If we take $k=p_{1}$, and if $C_{2}$ and $C_{3}$ are chosen coincident, Theorem II gives the following theorem, which is due to Bôcher:*

THEOREM III. If the roots of a binary form $f_{1}$ of degree $p_{1}$ lie in a closed region $T_{1}$ and if the roots of a second binary form $f_{2}$ of degree $p_{2}$ lie in a second closed region $T_{2}$ which has no point in common with $T_{1}$, and if these two regions are bounded by arcs of circles each one of which circles separates the interior of $T_{1}$ from the interior of $T_{2}$, then the jacobian of $f_{1}$ and $f_{2}$ has just $p_{1}-1$ roots in $T_{1}$ and $p_{2}-1$ roots in $T_{2} . \dagger$

* As Professor Curtiss pointed out to me, the statement given by Bôcher (l. c., p. 478) is not quite accurate. This inaccuracy has been here corrected.

$\dagger$ There have recently been published two results which are special cases of Theorem III, although the authors were apparently not aware of the fact.

See $\mathrm{L}$. R. Ford, On the roots of a derivative of a rational function, P r o c e e d in $\mathrm{gs}$ of the Edinburgh Mathematical Society, vol. 33 (1915). Several of Ford's results are generalized in the present paper.

See also B. Gonggrÿp, Quelques théorèmes, etc., Liouville's Journal, ser. 7, vol. 1 (1915), p. 360. Compare the former reference to Laguerre. 


\section{PART II}

All the theorems concerning jacobians which were proved by Bôcher, as well as the theorems of the present paper can be immediately applied to the roots of the derivative of a rational function.

Let us take any rational function not a constant, $f(z)=u(z) / v(z)$, and suppose (as we can do with no loss of generality) that $u$ and $v$ have no common factor containing $z$. Introduce homogeneous coördinates, setting $z=z_{1} / z_{2}$, and multiply the numerator and denominator of $f$ by $z_{2}^{n}$, where $n$ is the degree of $f:^{*}$

$$
f(z)=\frac{z_{2}^{n} u\left(z_{1} / z_{2}\right)}{z_{2}^{n} v\left(z_{1} / z_{2}\right)}=\frac{f_{1}\left(z_{1}, z_{2}\right)}{f_{2}\left(z_{1}, z_{2}\right)} .
$$

If we express $f^{\prime}(z)$, the derivative of $f(z)$, in terms of $J$, the jacobian of $f_{1}$ and $f_{2}$, we find

$$
f^{\prime}(z)=\frac{J}{n}\left(\frac{z_{2}}{f_{2}}\right)^{2} .
$$

From this relation it follows that the roots of $f^{\prime}$ are the roots of $J$ and a double root at infinity, except that when one of these points is also a pole of $f$ it cannot be a root of $f^{\prime}$.

We shall not attempt to carry over all the results concerning the roots of the jacobian to the corresponding results for the derivative of a rational function. We merely give a few examples by way of illustration. $\dagger$ The following theorem is a direct application of Theorem III.

If $f(z)$ is a rational function of degree $n$ whose roots lie in a closed region $T_{1}$ and whose poles lie in a second closed region $T_{2}$ which has no point in common with $T_{1}$, and if these two regions are bounded by arcs of circles each one of which circles separates the interior of $T_{1}$ from the interior of $T_{2}$; then all the roots of the derivative of $f(z)$ lie in $T_{1}$ and $T_{2}$, except that there are two additional roots at infinity if $f(z)$ has no pole there. Except for these two possible roots, there are just $n-1$ roots of $f^{\prime}(z)$ in $T_{1}$, and if $f(z)$ has no multiple pole there are just $n-1$ roots of $f^{\prime}(z)$ in $T_{2}$.†

* The degree of a rational function is the greater of the degrees of its numerator and denominator, or the common degree if the numerator and denominator have the same degree.

† Essentially the following theorem is given by Bôcher (l. c., p. 479): "If $f_{1}$ and $f_{2}$ are two forms and if all the roots of each form either lie on a circle $C$ or are situated in pairs of points inverse with respect to $C$, then all the roots of the jacobian of $f_{1}$ and $f_{2}$ also lie on $C$ or are situated in pairs of points inverse with respect to $C$. On any arc of $C$ bounded by roots of $f_{1}$ (or of $f_{2}$ ) and containing no root of either form there is at least one root of the jacobian."

It is true that the force at any point of $C$ (when the statical system is set up). is in direction tangent to $C$. Hence, if there are two circles $C$ of the theorem stated in this footnote, their intersection must be a root of the jacobian or a root of one of the ground forms.

Both of these theorems can evidently be extended to the derivative of a rational function.

‡ This is a generalization of the well-known theorem of Lucas that " the roots of the deriva- 
We can immediately obtain an upper bound for the moduli of the finite roots of the derivative of a rational function. Suppose $f$ to have $m_{1}$ finite roots (or poles) and $m_{2}$ finite poles (or roots), $m_{1}>m_{2}$. It follows from the corollary to Theorem II that if a circle whose radius is $a$ includes all the finite roots and poles of $f$, then a concentric circle of radius $a\left(m_{1}+m_{2}\right) /\left(m_{1}-m_{2}\right)$ includes all the finite roots of $f^{\prime}$.

Cambridae, Mass. January, 1918

tive of a polynomial lie within or on the boundary of the smallest convex polygon enclosing the roots of the original polynomial."

As Bôcher points out, Theorem III is also a generalization of Lucas's theorem.

The following theorem is a corollary of Theorem II: If $f(z)$ is a polynomial of degree $n$ which has a $k$-fold root at $P$, and if a circle whose center is $P$ and radius a includes no root of $f$ other than $P$, then the circle whose center is $P$ and radius ak / $n$ includes no root of $f^{\prime}(z)$ other than $P$. 